\title{
Utility of echocardiography in predicting mortality in infants with severe bronchopulmonary dysplasia
}

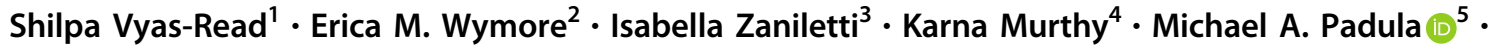 \\ William E. Truog ${ }^{6} \cdot$ William A. Engle ${ }^{7} \cdot{\text { Rashmin C. Savani } \mathbb{B}^{8} \cdot \text { Sushmita Yallapragada }^{8} \cdot \text { J. Wells Logan }}^{9}$. \\ Huayan Zhang ${ }^{5}$. Erik B. Hysinger ${ }^{10}$. Theresa R. Grover ${ }^{2}$ - Girija Natarajan ${ }^{11}$ - Leif D. Nelin ${ }^{9}$. Nicolas F. M. Porta $\mathbb{D}^{4}$. \\ Karin P. Potoka ${ }^{12} \cdot$ Robert DiGeronimo $^{13} \cdot$ Joanne M. Lagatta ${ }^{14} \cdot$ on behalf of the Children's Hospitals Neonatal \\ Consortium Severe BPD Focus Group
}

Received: 27 March 2019 / Revised: 9 September 2019 / Accepted: 18 September 2019 / Published online: 30 September 2019

(c) The Author(s), under exclusive licence to Springer Nature America, Inc. 2019

\begin{abstract}
Objective To determine the relationship between interventricular septal position (SP) and right ventricular systolic pressure (RVSP) and mortality in infants with severe BPD (sBPD).

Study design Infants with SBPD in the Children's Hospitals Neonatal Database who had echocardiograms 34-44 weeks' postmenstrual age (PMA) were included. SP and RVSP were categorized normal, abnormal (flattened/bowed SP or RVSP > $40 \mathrm{mmHg}$ ) or missing.

Results Of 1157 infants, 115 infants (10\%) died. Abnormal SP or RVSP increased mortality (SP 19\% vs. 8\% normal/ missing, RVSP $20 \%$ vs. $9 \%$ normal/missing, both $p<0.01$ ) in unadjusted and multivariable models, adjusted for significant covariates (SP OR 1.9, 95\% CI 1.2-3.0; RVSP OR 2.2, 95\% CI 1.1-4.7). Abnormal parameters had high specificity (SP 82\%; RVSP 94\%), and negative predictive value (SP 94\%, NPV 91\%) for mortality.

Conclusions Abnormal SP or RVSP is independently associated with mortality in SBPD infants. Negative predictive values distinguish infants most likely to survive.
\end{abstract}

These authors contributed equally: Shilpa Vyas-Read, Erica M. Wymore

Shilpa Vyas-Read

svyasre@emory.edu

1 Children's Healthcare of Atlanta, Emory University School of Medicine, Atlanta, GA, USA

2 Children's Hospital Colorado, University of Colorado School of Medicine, Aurora, CO, USA

3 Children's Hospital Association, Lenexa, KS, USA

4 Ann and Robert H. Lurie Children's Hospital of Chicago and Department of Pediatrics, Feinberg School of Medicine, Northwestern University, Chicago, IL, USA

5 Children's Hospital of Philadelphia, University of Pennsylvania Perelman School of Medicine, Philadelphia, PA, USA

6 Children's Mercy Hospital, University of Missouri, Kansas City, MO, USA

7 Riley Hospital for Children, Department of Pediatrics, Indiana

\section{Introduction}

Severe bronchopulmonary dysplasia (sBPD) affects approximately $20 \%$ of extremely low-birthweight infants [1]. Pulmonary arterial hypertension (PH) can occur in

University School of Medicine, Indianapolis, IN, USA

8 University of Texas Southwestern Medical Center, Dallas, TX, USA

9 Nationwide Children's Hospital, The Ohio State University, Columbus, OH, USA

10 Cincinnati Children's Hospital Medical Center, Department of Pediatrics, Cincinnati, OH, USA

11 Children's Hospital of Michigan, Wayne State University, Detroit, MI, USA

12 Department of Pediatrics, Division of Newborn Medicine, Children's Hospital of Pittsburgh of UPMC, Pittsburgh, PA, USA

13 Seattle Children's Hospital, University of Washington, Seattle, WA, USA

14 Children's Hospital of Wisconsin, Department of Pediatrics, Medical College of Wisconsin, Milwaukee, WI, USA 
$15-25 \%$ of infants with sBPD, and is associated with an increased risk of mortality [2-4]. Recognizing this concern, the American Heart Association recommends screening echocardiography to monitor for $\mathrm{PH}$ in infants with BPD [5]. However, echocardiographic parameters are limited by subjective, varied, or missing measures, as well as modest correlation with pulmonary arterial pressure measured via cardiac catheterization [6-9]. Further, screening for $\mathrm{PH}$ often varies from center to center. Understanding whether standard clinically used echocardiographic parameters of $\mathrm{PH}$ are associated with mortality would help to inform clinical practices with respect to screening infants with sBPD for PH.

Our objective was to determine whether interventricular septal position and right ventricular systolic pressure on echocardiography performed between 34 and 44 weeks' post-menstrual age (PMA) were associated with in-hospital mortality in infants with sBPD. We hypothesized that these measures could be used to predict inpatient mortality in a real-world clinical cohort.

\section{Methods}

We performed a secondary analysis of data from the Children's Hospitals Neonatal Database (CHND), which includes prospectively collected data on all infants admitted to participating Level IV neonatal intensive care units (NICUs) in the United States and Canada. Infant clinical data were collected by trained chart abstractors at each site, with periodic assessments of inter-rater agreement scores [10]. De-identified data analyses were approved by the Institutional Review Board of Stanley Manne Research Institute affiliated with the Ann and Robert H. Lurie Children's Hospital of Chicago. All participating centers obtained regulatory oversight to participate in the CHND registry.

We included infants born $<32$ weeks' gestation who were admitted to a participating NICU between 2010 and 2015, who had SBPD, and who had at least one echocardiogram between 34 and 44 weeks' PMA. sBPD was defined as receiving positive pressure ventilation, $>2 \mathrm{~L} / \mathrm{min}$ flow by nasal cannula, or fractional inspired oxygen ( $\mathrm{FiO} 2)$ $>0.3$ at or after 36 weeks' PMA [2, 10, 11]. Infants were excluded if they died prior to 36 weeks' PMA, were transferred out of the participating hospital before discharge, or if they had major congenital anomalies. Infants with an isolated atrial septal defect, ventricular septal defect, or patent ductus arteriosus were retained in the study.

The primary outcome was mortality between 36 weeks' PMA and discharge from a CHND hospital. Primary exposures of interest were echocardiographic measures of interventricular septal position (SP) and right ventricular systolic pressure (RVSP) recorded between 34 and 44 weeks' PMA. SP was defined as abnormal if there was documentation of septal position being flattened or bowed to the left. RVSP was categorized as abnormal if the result was $>40 \mathrm{mmHg}$; this cut-off was determined a priori corresponding to values $>40 \mathrm{mmHg}$ being more than half of the systemic blood pressure [7, 12, 13]. For infants with multiple echocardiograms, we examined all reports occurring between 34 and 44 weeks PMA; we categorized the infant as having an abnormal echocardiographic parameter if any available studies had an abnormal parameter. If no available echocardiography reports documented RVSP or SP, we categorized the data as missing and retained that infant in the dataset. Direction of flow across the patent ductus arteriosus (PDA) and the interatrial septum were not considered as exposures for this study because the PDA was closed and interatrial septal flow was minimal in nearly all subjects between 34 and 44 weeks' PMA. Instead, PDA ligation and interatrial communication were included as potential covariates.

We included other clinical covariates available in the CHND that we hypothesized might confound the relationship between echocardiographic parameters and mortality. Maternal characteristics included were race, diabetes, hypertension, chorioamnionitis, multiple gestation, and receipt of antenatal steroids. Neonatal characteristics included gestational age, birthweight, small for gestational age (defined as sex-specific $\leq 10$ th percentile of birthweight for gestational age) [14], duration of mechanical ventilation in the CHND nursery, receipt of mechanical ventilation at 36 weeks' PMA, receipt of systemic corticosteroids, bloodstream infections, atrial septal communications, or PDA ligation. A respiratory severity score was also calculated as a product of the mean airway pressure and the fractional oxygen requirement $\left(\mathrm{MAP} \times \mathrm{FiO}_{2}\right)$ at 36 weeks' PMA and compared between groups (see Table 1). Bloodstream infections were defined as any confirmed bacterial or fungal organisms recovered from a blood culture during the hospital admission. Necrotizing enterocolitis was defined as any of the following conditions: (1) Surgical NEC; (2) NEC at post-mortem examination or (3) if both clinical symptoms (bilious aspirate or emesis, abdominal distention, and occult/gross blood in stool) and radiographic findings (pneumotosis intestinalis, hepatobiliary gas or pneumoperitonem) were present. These findings correspond to a NEC Stage IIb or higher. Airway comorbidities were defined as laryngeal, tracheal, or bronchial malacia, laryngeal or tracheal stenosis, or vocal cord paralysis or paresis.

\section{Statistical analysis}

We first assessed the proportion of echocardiographic parameters that were normal, abnormal and missing in the 
Table 1 Baseline cohort demographics by septal position and right ventricular systolic pressure

\begin{tabular}{|c|c|c|c|c|}
\hline Septal position variables & Septum abnormal & $\begin{array}{l}\text { Septum data } \\
\text { missing }\end{array}$ & Septum normal & $p$ value \\
\hline$N(\%)$ & $227(20)$ & $314(27)$ & $616(53)$ & \\
\hline Gestational age (weeks) median (IQR) & $25(24,26)$ & $25(24,27)$ & $25(24,27)$ & 0.002 \\
\hline Admission PMA (weeks) median (IQR) & $29(26,36)$ & $28(26,31)$ & $29(26,33)$ & 0.003 \\
\hline $\begin{array}{l}\text { PMA at echocardiogram (wks) } \\
\text { median (IQR) }\end{array}$ & $31(27,40)$ & $30(27,36)$ & $31(27,37)$ & 0.017 \\
\hline Birthweight (g) median (IQR) & $680(580,790)$ & $740(610,910)$ & $766(640,940)$ & 0.000 \\
\hline$\leq 10$ th centile, $n(\%)$ & $59(26)$ & $55(18)$ & $112(18)$ & 0.023 \\
\hline Maternal race, $n(\%)$ & & & & 0.031 \\
\hline White & $105(46)$ & $171(55)$ & $325(53)$ & \\
\hline Black & $95(42)$ & $93(30)$ & $216(35)$ & \\
\hline Other & $24(11)$ & $39(12)$ & $67(11)$ & \\
\hline Unknown & $3(1)$ & $11(4)$ & $8(1)$ & \\
\hline Ventilator days median (IQR) & $55(28,92)$ & $46.5(19,83)$ & $42(20,71)$ & 0.002 \\
\hline Ventilator at 36 weeks' PMA, $n(\%)$ & $120(53)$ & $127(41)$ & $233(38)$ & 0.000 \\
\hline $\begin{array}{l}\text { MAP } \times \text { FiO2 @ } 36 \text { weeks' PMA for } \\
\text { ventilated patients median (IQR) }\end{array}$ & $4(3,7)$ & $2(0,5)$ & $3(2,6)$ & 0.000 \\
\hline Systemic steroids, $n(\%)$ & $113(50)$ & $91(29)$ & $276(45)$ & 0.000 \\
\hline Fundoplication, $n(\%)$ & $37(16)$ & $20(6)$ & $61(10)$ & 0.001 \\
\hline Bloodstream infection, $n(\%)$ & $69(30)$ & $92(29)$ & $141(23)$ & 0.028 \\
\hline Atrial septal defect, $n(\%)$ & $62(28)$ & $28(9)$ & $62(10)$ & 0.000 \\
\hline PMA at PDA Ligation median (IQR) & $32(29,39)$ & $30(28,33)$ & $30(28,32)$ & 0.001 \\
\hline RVSP variables & RVSP > 40 mmHg & RVSP missing & RVSP $\leq 40 \mathrm{mmHg}$ & $p$ value \\
\hline$N(\%)$ & $84(7)$ & $843(73)$ & $230(20)$ & \\
\hline Gestational age (weeks) median (IQR) & $25(24,26)$ & $25(24,27)$ & $25(24,26)$ & 0.000 \\
\hline $\begin{array}{l}\text { PMA at echocardiogram (wks) } \\
\text { median (IQR) }\end{array}$ & $34(27,40)$ & $30(27,37)$ & $30(26,37)$ & 0.020 \\
\hline Birthweight (g) median (IQR) & $668(570,826)$ & $760(630,941)$ & $703(585,812)$ & 0.000 \\
\hline Male gender, $n(\%)$ & $39(46)$ & $530(63)$ & $133(58)$ & 0.008 \\
\hline \multicolumn{5}{|l|}{ Maternal race, $n(\%)$} \\
\hline White & $38(45)$ & $460(55)$ & $103(45)$ & 0.009 \\
\hline Black & $35(42)$ & $280(33)$ & $89(39)$ & \\
\hline Other & $10(12)$ & $83(10)$ & $37(16)$ & \\
\hline Unknown & $1(1)$ & $20(2)$ & $1(0)$ & \\
\hline Multiple births, $n(\%)$ & $12(14)$ & $210(25)$ & $52(23)$ & 0.017 \\
\hline Atrial septal defect, $n(\%)$ & $16(19)$ & $99(12)$ & $39(17)$ & 0.033 \\
\hline PMA at PDA ligation median (IQR) & $37(30,39)$ & $30(28,33)$ & $29(28,31)$ & 0.000 \\
\hline
\end{tabular}

Baseline cohort demographics by septal flattening and right ventricular systolic pressure measurements

Baseline demographic and clinical variables were compared between septal flattening categories of abnormal position (flattened or bowed), septal position data missing, and normal position; and right ventricular systolic pressure (RVSP) categories of RVSP abnormal (>40 mmHg), RVSP data missing, or RVSP normal ( $\leq 40 \mathrm{mmHg})$. Chi-square tests were used to compare proportions and $t$ tests or nonparametric tests were used to compare medians of continuous variables

Septal position did not differ between groups with respect to male gender, maternal chorioamnionitis, maternal diabetes, maternal hypertension, multiple births, antenatal steroids, airway comorbidity, tracheostomy, gastrostomy, surgical necrotizing enterocolitis, ventricular septal defect, and PDA ligation $(p>0.05)$. Median admission PMA and ventilator days did not differ between RVSP groups. The proportion of infants with birthweight $<10$ th\%, with maternal chorioamnionitis, diabetes, or hypertension, receiving antenatal steroids, on the ventilator at 36 weeks' PMA, with airway comorbidities, with tracheostomy, with surgical necrotizing enterocolitis, with gastrostomy or fundoplication, with bloodstream infections, with ventricular septal defects, or with PDA ligations did not differ between RVSP groups $(p \geq 0.05)$

$P M A$ post-menstrual age, $M A P$ mean airway pressure, $P D A$ patent ductus arteriosus 
study cohort. We compared the clinical characteristics by SP and RVSP groups of normal, abnormal and missing, using chi-squared or Fisher's exact tests for differences in proportion and Kruskal-Wallis nonparametric tests for differences of medians. For the unadjusted association between echocardiographic parameters and mortality, we calculated the sensitivity, specificity, positive and negative predictive value, and relative risk. Next, all covariates with bivariate association of $p<0.05$ were entered into multilevel multivariable logistic regression models with center as a random intercept. Because we assumed the echocardiographic exposure variables to be collinear, we ran two separate models, one with septal position alone as the exposure, and one with RVSP alone as the exposure. For each model, abnormal and missing echocardiographic parameters were each tested against a referent group of infants with normal echocardiographic parameters. Statistical significance was defined as $p$ value $<0.05$. SAS v 9.2 (SAS Institute, Cary, North Carolina) was used for analyses.

\section{Results}

The database included 12,221 infants born $<32$ weeks' gestation who were alive at 36 weeks' PMA and who did not have congenital anomalies. Of those, 2806 infants had sBPD and $1412(50 \%)$ infants with sBPD had one or more echocardiograms between 34 and 44 weeks' PMA. We excluded 255 infants who were transferred to another hospital before discharge. This resulted in a study cohort of 1157 infants; 1042 (90\%) survived to hospital discharge, while 115 (10\%) died. Infants who died had a later PMA at admission to the NICU (median 31 days [IQR 27-38] vs. 28 [IQR 26-33], $p<0.001$ ), lower birthweight (median $685 \mathrm{~g}$ [IQR 560-840] vs. $748 \mathrm{~g}$ [IQR 620-910], $p=0.001$ ), were on the ventilator longer (median 102 days [IQR 51-144] vs. 42 days [IQR 19-71], $p<0.001$ ), and were more likely to have received systemic corticosteroids $(69 \%$ vs. $38 \%, p<$ 0.001 ) or to have received a diagnosis of necrotizing enterocolitis $(22 \%$ vs. $10 \%, p=0.001)$ than infants who survived. The cause of death in our cohort was primarily respiratory failure $(67 \%, n=77 / 115)$, followed by multiorgan system failure $(16 \%, n=18 / 115)$. Forty-six infants $(60 \%, n=46 / 77)$ who died of respiratory causes also had a clinical diagnosis of pulmonary hypertension. Less common causes of death were intra-abdominal catastrophe (4\%), central nervous system injury (3\%), infection (3\%), renal failure (3\%), and other causes (4\%). Infants with abnormal septal position and RVSP had a median of 2 echocardiograms, and infants with normal or missing parameters had a median of one echocardiogram during their neonatal hospitalization. As shown in Table 1, infants with abnormal RVSP measurements had echocardiograms performed at a slightly later PMA those with normal measurements (34 weeks vs. 30 weeks), and the median PMA of echocardiogram attainment in infants with abnormal and normal septal position was 31 weeks.

Septal position (SP) was categorized as normal in 616 (53\%) infants, abnormal in 227 (20\%) infants, and missing in $314(27 \%)$ of infants. Table 1 (top) compares characteristics of infants by SP. SP groups differed with respect to gestational age and birthweight, age at referral to CHND NICU, birthweight $<10$ th percentile for gestational age, maternal race, duration of mechanical ventilation, respiratory severity score at 36 weeks' PMA, receipt of systemic corticosteroids, bloodstream infections, and atrial septal defects. The proportion of infants receiving PDA ligation was similar between groups but the PMA at PDA ligation was later in infants with abnormal SP. Infants with missing SP had clinical characteristics that were similar to those of infants with normal position. RVSP was categorized as normal in $230(20 \%)$ infants, abnormal in $84(7 \%)$, and missing in $843(73 \%)$. Table 1 (bottom) compares characteristics of infants by RVSP. RVSP categories differed in categories of birthweight, sex, maternal race, singleton birth and atrial septal defects. Rates of PDA ligation were similar between groups but the median PMA at ligation was later in infants with abnormal RVSP. Infants with missing RVSP had the largest birthweight, the lowest receipt of postnatal systemic steroids, and the lowest proportion of atrial septal defects.

Abnormal SP and RVSP were each associated with higher odds of mortality (Table 2, top). In unadjusted analysis, abnormal SP was associated with 2.6-fold increase in mortality, and after adjustment for significant covariates, SP was associated with 1.9-times the odds of mortality, when compared with infants with normal SP $(p=0.01)$. However, infants with missing SP did not have significantly different odds of mortality from those with normal SP. Receiving ventilation at 36 weeks' PMA, having a bloodstream infection, receiving postnatal steroids, and being admitted at a later PMA were also significant risk factors for mortality in both unadjusted and adjusted SP models. Similarly, in both unadjusted models and adjusted models, abnormal RVSP was associated with a 2.2 -fold increase in mortality $(p=0.02)$, whereas infants with missing RVSP data had mortality rates that were similar to infants with normal RVSP values (Table 2, bottom). Ventilation at 36 wks' PMA, bloodstream infection, postnatal steroid use, and admission PMA remained significantly associated with mortality in adjusted RVSP models. Whereas atrial septal defects marginally increased the odds of mortality in SP models, the presence of an interatrial communication significantly increased mortality by 1.9 -fold in RVSP models.

Overall, infants with abnormal septal position had a mortality rate that was $19 \%$ compared with $8 \%$ of infants 
Table 2 Models of clinical and echocardiographic variables on mortality

\begin{tabular}{lllll}
\hline Variable & $\begin{array}{l}\text { Unadjusted } \\
\text { odds ratio }\end{array}$ & $95 \%$ CI & $\begin{array}{l}\text { Adjusted } \\
\text { odds ratio }\end{array}$ & 95\% CI \\
\hline Septal position & & & & \\
$\quad$ Abnormal vs. Normal* & 2.6 & $1.7-4.1$ & 1.9 & $1.2-3.0$ \\
$\quad$ Missing vs. Normal & 0.9 & $0.5-1.4$ & 0.9 & $0.5-1.5$ \\
Gestational age & 1.0 & $0.9-1.1$ & 1.0 & $0.9-1.1$ \\
Ventilated at 36 weeks' PMA* & 6.7 & $4.2-10.8$ & 5.3 & $3.2-8.6$ \\
Atrial septal defect & 1.7 & $1.1-2.8$ & 1.6 & $1.0-2.8$ \\
Bloodstream infection* & 1.7 & $1.1-2.5$ & 1.8 & $1.1-2.8$ \\
Postnatal steroids* & 3.9 & $1.9-8.1$ & 2.4 & $1.1-5.2$ \\
PMA at admission* (per postnatal week) & 1.1 & $1.0-1.1$ & 1.1 & $1.0-1.1$ \\
RVSP & & & & \\
$\quad$ Abnormal vs. normal* & 2.2 & $1.1-4.3$ & 2.2 & $1.1-4.7$ \\
$\quad$ Missing vs. normal & 0.8 & $0.5-1.3$ & 0.9 & $0.6-1.6$ \\
Gestational age & 1.0 & $0.9-1.1$ & 1.0 & $0.9-1.1$ \\
Ventilated at 36 weeks' PMA* & 6.7 & $4.2-10.8$ & 5.7 & $3.5-9.4$ \\
Atrial septal defect* & 1.7 & $1.1-2.8$ & 1.9 & $1.1-3.2$ \\
Bloodstream infection* & 1.7 & $1.1-2.5$ & 1.7 & $1.1-2.8$ \\
Postnatal steroids* & 3.9 & $1.9-8.1$ & 2.4 & $1.1-5.2$ \\
PMA at admission* (per postnatal week) & 1.1 & $1.0-1.1$ & 1.1 & $1.0-1.1$ \\
\hline Multivariale models were constucted & & & \\
\hline
\end{tabular}

Multivariable models were constructed to evaluate the effect of septal position alone, and right ventricular systolic pressure (RVSP) alone on mortality. Significant clinical covariates, including gestational age, the need for ventilation at 36 weeks' postmenstrual age, the presence of an atrial septal defect, the diagnosis of a bloodstream infection, the receipt of postnatal steroids and the postmenstrual age in weeks at the time of admission, were controlled in each model. Odds ratios and 95\% confidence intervals were calculated using logistic regression

$* p<0.05$ in multivariable models with normal SP, and $8 \%$ for infants with normal/missing data (abnormal SP $n=43 / 227$ vs. normal SP $n=50 / 616$ or normal/missing SP $n=72 / 930)$. Mortality for infants with abnormal RVSP was $20 \%(n=17 / 84)$ compared with $10 \%$ for infants with normal data $(n=24 / 230)$ and $9 \%$ for infant with normal/missing data $(n=98 / 1073)$.

Test characteristics of the abnormal echocardiographic parameters are shown in Table 3. When comparing infants with abnormal SP or RVSP to those with normal parameters, these parameters were highly specific (82\% and $94 \%$, respectively) but poorly sensitive (37\% and 15\%) markers of mortality. The negative predictive value was over $90 \%$ for each parameter. This pattern was nearly identical when comparing abnormal SP or RVSP to infants either a normal or missing parameter.

\section{Discussion}

In this multicenter clinical cohort of infants with SBPD, we found that septal position and RVSP $>40 \mathrm{mmHg}$ were significantly associated with in-hospital mortality after adjusting for significant clinical characteristics. We also found that infants with missing documentation of RVSP
Table 3 Sensitivity analyses for mortality by echocardiographic parameter

\begin{tabular}{lllll}
\hline Echocardiographic variable & Sensitivity & Specificity & PPV & NPV \\
\hline Septal position & & & & \\
$\quad$ Abnormal vs. normal & $46 \%$ & $75 \%$ & $19 \%$ & $92 \%$ \\
$\quad$ Abnormal vs. normal/missing & $37 \%$ & $82 \%$ & $19 \%$ & $92 \%$ \\
RVSP $>40 \mathrm{mmHg}$ & & & & \\
$\quad$ Abnormal vs. normal & $41 \%$ & $75 \%$ & $20 \%$ & $90 \%$ \\
$\quad$ Abnormal vs. normal/missing & $15 \%$ & $94 \%$ & $20 \%$ & $91 \%$ \\
\hline
\end{tabular}

Mortality rates for infants with abnormal SP were $19 \%$ compared with $8 \%$ for infants with normal or normal/missing data. Mortality rates for infants with abnormal RVSP were $20 \%$ compared with $9-10 \%$ for infants with normal or normal/missing data. Test characteristics of sensitivity, specificity, positive-predictive value (PPV) and negative predictive value (NPV) are shown

and/or septal position had clinical and mortality outcomes similar to those with documented normal echocardiographic parameters.

Pulmonary hypertension is a significant contributor to morbidity and mortality in premature infants with BPD [2, 15-18]. In 2015, the American Heart Association recommended echocardiographic screening of infants with 
moderate or severe BPD beginning at 36 weeks PMA with serial echocardiography thereafter [5]. Compared to cardiac catheterization, echocardiographic screening for $\mathrm{PH}$ has been limited in its ability to detect and grade the severity of pulmonary hypertension [7]. We found that infants with documented abnormal RVSP or septal position had approximately double the odds of mortality, compared to infants with normal echocardiographic parameters. This association persisted even after adjusting for other significant predictors of mortality including gestational age, receipt of mechanical ventilation at 36 weeks' PMA, atrial septal defect, bloodstream infection, postnatal corticosteroid use, and PMA at admission to the participating center. These results demonstrate that even among sick neonates with severe BPD, abnormal SP and RVSP are independent predictors of mortality. In that context, our results demonstrating the potential impact of real-world echocardiographic data provide further support for adherence to the new guidelines.

When we compared infants with missing echocardiographic data to infants with normal parameters for septal position, we found that infants with missing data had an odds of mortality that was similar to infants with normal echocardiographic parameters. Mourani et al. found that the sensitivity and positive-predictive value of echocardiographic parameters for the detection of $\mathrm{PH}$ is enhanced when tricuspid regurgitant jet velocity can be measured as a proxy for pulmonary artery pressure [7]. Unfortunately, several studies have noted the challenge of obtaining tricuspid regurgitant jet velocity measurements, even when echocardiography is being performed in accordance with prespecified research protocols and on older pediatric patients $[19,20]$. Indeed, in our cohort, tricuspid regurgitant jet velocity and resultant right ventricular systolic pressure was recorded in only $27 \%$ of echocardiographic reports, highlighting the practical limitations of obtaining this important variable at the bedside. Septal position was more commonly documented in our cohort, with $73 \%$ of echocardiographic reports having documentation of septal position. Assignment of septal position may be subject to considerable subjectivity and interobserver variability [21]. More quantitative measures of septal position, such as eccentricity index, are now being investigated in infants with BPD and hold promise as a means of standardizing the echocardiographic approach [19, 22]. Nonetheless, our finding that infants with missing echocardiographic data had mortality rates similar to infants with documented normal echocardiographic parameters suggests that the inherent difficulties of obtaining standard echocardiographic measurements should not prevent efforts at screening. Further, echocardiography correctly identified infants who were likely to survive, as shown by the high specificity and negative predictive values of both septal flattening and RVSP in our study. As such, septal position and RVSP measures on echocardiography between 34 and 44 weeks' PMA can be helpful in delineating risk status for in-hospital mortality in infants with sBPD.

Surprisingly, only half of our initial cohort of infants with severe BPD had screening echocardiography between 34 and 44 weeks' PMA. This finding is in keeping with recent surveys of neonatal members of the American Academy of Pediatrics. This survey found that $83 \%$ of physicians would obtain an echocardiogram at 36 weeks' corrected gestational age for infants with moderate or severe BPD, but only $46 \%$ of respondents had a formal screening program in place at their institution that facilitated the implementation of these guidelines [23]. Our study cohort occurred prior to the publication of the recent American Heart Association screening guidelines; future work, including our observations in this study, should serve to increase the rate of screening for $\mathrm{PH}$ in infants with BPD. Quality initiatives to standardize the approach to $\mathrm{PH}$ screening in infants with BPD across sites are now being developed. Even as screening efforts advance, we acknowledge that data are limited on whether early detection and pharmacologic treatment of pulmonary hypertension in infants with severe BPD improves survival. Retrospective evaluations of this association may be plagued with concerns about confounding by indication, and the feasibility and cost of randomized controlled trials can be prohibitive. Additionally, the natural course of milder forms of pulmonary hypertension is to resolve without pharmacologic therapy [24, 25]. However, identifying the patients at risk for poor outcomes is essential in the neonatal period to allow appropriate risk categorization and followup after hospital discharge. Overall, one in four infants with severe BPD may develop pulmonary hypertension, but 41 $-62 \%$ will be diagnosed after hospital discharge and will experience morbidity and mortality in the first year of life as a result of their diagnosis $[1,2,24,26]$. As such, even while the efficacy of pharmacologic therapy for pulmonary hypertension is being evaluated, echocardiographic screening for infants with severe BPD may allow proven interventions such as aggressive management of respiratory acidosis, prevention of intermittent hypoxia, targeting of optimal nutrition and feeding modes, and engagement of multidisciplinary care to be employed early and aggressively in order to improve outcomes for high-risk infants [1, 27-31].

Our study has important limitations. Our cohort was derived from a referral-based, quaternary care population and may not be generalizable to other cohorts. We further recognize that the infants in our cohort had severe BPD, and although controlling for the need for mechanical ventilation at 36 weeks' post-menstrual age may have adjusted for the 
sickest patients, it is possible that associations between echocardiographic parameters and mortality may differ in infants with less severe lung disease. The echocardiograms in our study were performed at clinical discretion, which may lead to some ascertainment and selection bias in our sample.

In conclusion, we have shown that abnormalities in echocardiographic parameters, such as septal flattening and RVSP, are associated with nearly a two-fold increase in the odds of mortality prior to hospital discharge in infants with severe BPD. This association persists after controlling for important clinical variables. Further, infants with normal parameters are more likely to survive their initial hospitalization than those with abnormal values in our cohort. These findings support the recommendation to implement pulmonary hypertension screening for infants with severe BPD despite the known limitations of echocardiography in a neonatal population. Future quality improvement initiatives that define the timing and standardize the optimal approach to echocardiography in this high-risk population are warranted.

\section{Code availability}

The computer code used to generate statistical analyses may be made available upon request to the Children's Hospitals Neonatal Consortium.

Funding The Children's Hospitals Neonatal Consortium (501-c3 organization) supported the statistical analyses presented in this manuscript.

Author contributions SV-R, EMW, KM, JML: Research question and design, data analysis and interpretation, draft preparation, editing, and revision of the manuscript. IZ: Statistical analysis of the manuscript. MAP, WET, WAE, RCS, SY, JWL, HZ, EBH, TRG, GN, LDN, NFMP, KPP, RD: Data interpretation, editing and revision of the manuscript.

\section{Compliance with ethical standards}

Conflict of interest The authors declare that they have no conflict of interest.

Publisher's note Springer Nature remains neutral with regard to jurisdictional claims in published maps and institutional affiliations.

\section{References}

1. Abman SH, Collaco JM, Shepherd EG, Keszler M, CuevasGuaman M, Welty SE. et al. Interdisciplinary care of Children with Severe Bronchopulmonary Dysplasia. J Pediatr. 2017;181: 12-28.e1.

2. Lagatta JM, Hysinger EB, Zaniletti I, Wymore EM, Vyas-Read S, Yallapragada S, et al. The impact of pulmonary hypertension in preterm infants with severe bronchopulmonary dysplasia through 1 year. J Pediatr. 2018. https://doi.org/10.1016/j.jpeds. 2018.07.035.

3. Berkelhamer SK, Mestan KK, Steinhorn RH. Pulmonary hypertension in bronchopulmonary dysplasia. Semin Perinatol. 2013; 37:124-131.

4. Slaughter JL, Pakrashi T, Jones DE, South AP, Shah TA. Echocardiographic detection of pulmonary hypertension in extremely low birth weight infants with bronchopulmonary dysplasia requiring prolonged positive pressure ventilation. J Perinatol. 2011;31:635-640.

5. Abman SH, Hansmann G, Archer SL, Ivy DD, Adatia I, Chung WK, et al. Pediatric pulmonary hypertension: guidelines from the American Heart Association and American Thoracic Society. Circulation. 2015;132:2037-2099.

6. Weismann CG, Asnes JD, Bazzy-Asaad A, Tolomeo C, Ehrenkranz RA, Bizzarro MJ. Pulmonary hypertension in preterm infants: results of a prospective screening program. J Perinatol. 2017;37:572-577.

7. Mourani PM, Sontag MK, Younoszai A, Ivy DD, Abman SH. Clinical utility of echocardiography for the diagnosis and management of pulmonary vascular disease in young children with chronic lung disease. Pediatrics. 2008;121: 317-325.

8. McCrary AW, Barker PCA, Torok RD, Spears TG, Li JS, Hornik $\mathrm{CP}$, et al. Agreement of an echocardiogram-based diagnosis of pulmonary hypertension in infants at risk for bronchopulmonary dysplasia among masked reviewers. J Perinatol. 2018. https://doi. org/10.1038/s41372-018-0277-6.

9. Carlton EF, Sontag MK, Younoszai A, DiMaria MV, Miller JI, Poindexter BB, et al. Reliability of echocardiographic indicators of pulmonary vascular disease in preterm infants at risk for bronchopulmonary dysplasia. J Pediatr. 2017. https://doi.org/10. 1016/j.jpeds.2017.03.027.

10. Murthy K, Porta NFM, Lagatta JM, Zaniletti I, Truog WE, Grover $\mathrm{TR}$, et al. Inter-center variation in death or tracheostomy placement in infants with severe bronchopulmonary dysplasia. J Perinatol. 2017;37:723-727.

11. Hysinger EB, Friedman NL, Padula MA, Shinohara RT, Zhang H, Panitch HB, et al. Tracheobronchomalacia is associated with increased morbidity in bronchopulmonary dysplasia. Ann Am Thorac Soc. 2017;14. https://doi.org/10.1513/AnnalsATS. 201702-178OC.

12. Mirza H, Ziegler J, Ford S, Padbury J, Tucker R, Laptook A. Pulmonary hypertension in preterm infants: prevalence and association with bronchopulmonary dysplasia. J Pediatr. 2014;165: 909-14.e1.

13. del Cerro MJ, Sabate Rotes A, Carton A, Deiros L, Bret M, Cordeiro M, et al. Pulmonary hypertension in bronchopulmonary dysplasia: clinical findings, cardiovascular anomalies and outcomes. Pediatr Pulmonol. 2014;49:49-59.

14. Olsen IE, Groveman SA, Lawson ML, Clark RH, Zemel BS. New intrauterine growth curves based on United States data. Pediatrics. 2010;125:e214-24.

15. Al-Ghanem G, Shah P, Thomas S, Banfield L, Helou El S, Fusch C. et al. Bronchopulmonary dysplasia and pulmonary hypertension: a meta-analysis. J Perinatol. 2017;37:414-419.

16. Ali Z, Schmidt P, Dodd J, Jeppesen DL. Predictors of bronchopulmonary dysplasia and pulmonary hypertension in newborn children. Dan Med J. 2013;60:A4688.

17. Baker CD, Abman SH, Mourani PM. Pulmonary hypertension in preterm infants with bronchopulmonary dysplasia. Pediatr Allergy, Immunol, Pulmonol. 2014;27:8-16.

18. Murthy K, Savani RC, Lagatta JM, Zaniletti I, Wadhawan R, Truog W, et al. Predicting death or tracheostomy placement in infants with severe bronchopulmonary dysplasia. J Perinatol. 2014;34:543-548. 
19. Abraham S, Weismann CG. Left ventricular end-systolic eccentricity index for assessment of pulmonary hypertension in infants. Echocardiography. 2016;33:910-915.

20. Zivanovic S, Pushparajah K, Calvert S, Marlow N, Razavi R, Peacock JL. et al. Pulmonary artery pressures in school-age children born prematurely. J Pediatr. 2017;191:42-49.e3.

21. Watson T, McCracken CE, Slesnick T, Kanaan U, Border WL, Sachdeva R. Quantitative assessment of ventricular septal contour for estimation of right ventricular pressure. Echocardiography. 2016;33:444-9. quiz 443

22. McCrary AW, Malowitz JR, Hornick CP, Hill KD, Cotten CM, Tatum GH, et al. Differences in eccentricity index and systolicdiastolic ratio in extremely low-birth-weight infants with bronchopulmonary dysplasia at risk of pulmonary hypertension. Am J Perinatol. 2016;33:57-62.

23. Altit G, Lee HC, Hintz S, Tacy TA, Feinstein JA, Bhombal S. Practices surrounding pulmonary hypertension and bronchopulmonary dysplasia amongst neonatologists caring for premature infants. J Perinatol. 2018;38:361-367.

24. Mehler K, Udink Ten Cate FE, Keller T, Bangen U, Kribs A, Oberthuer A. An echocardiographic screening program helps to identify pulmonary hypertension in extremely low birthweight infants with and without bronchopulmonary dysplasia: a singlecenter experience. Neonatology. 2018;113:81-88.
25. An HS, Bae EJ, Kim GB, Kwon BS, Beak JS, Kim EK, et al. Pulmonary hypertension in preterm infants with bronchopulmonary dysplasia. Korean Circ J. 2010;40:131-136.

26. Khemani E, McElhinney DB, Rhein L, Andrade O, Lacro RV, Thomas KC, et al. Pulmonary artery hypertension in formerly premature infants with bronchopulmonary dysplasia: clinical features and outcomes in the surfactant era. Pediatrics. 2007;120:1260-1269.

27. Martin RJ, Di Fiore JM, Walsh MC. Hypoxic episodes in bronchopulmonary dysplasia. Clin Perinatol. 2015;42:825-838.

28. Kovesi T, Abdurahman A, Blayney M. Elevated carbon dioxide tension as a predictor of subsequent adverse events in infants with bronchopulmonary dysplasia. Lung. 2006;184:7-13.

29. Thome UH, Dreyhaupt J, Genzel-Boroviczeny O, Bohnhorst B, Schmid M, Fuchs $\mathrm{H}$, et al. Influence of PCO2 control on clinical and neurodevelopmental outcomes of extremely low birth weight infants. Neonatology. 2018;113:221-230.

30. Brown MK, Poeltler DM, Hassen KO, Lazarus DV, Brown VK, Stout JJ, et al. Incidence of hypocapnia, hypercapnia, and acidosis and the associated risk of adverse events in preterm neonates. Respir Care. 2018;63:943-949.

31. Krishnan U, Feinstein JA, Adatia I, Austin ED, Mullen MP, Hopper RK, et al. Evaluation and management of pulmonary hypertension in children with bronchopulmonary dysplasia. J Pediatr. 2017. https://doi.org/10.1016/j.jpeds.2017.05.029. 